\title{
LOUVA-A-DEUSES (Dictyoptera, Mantodea) DO NORDESTE BRASILEIRO
}

\author{
Vanine Haywanon Santos Maia ${ }^{1}$ e Freddy Ruben Bravo Quijano ${ }^{2}$; \\ 1. Bolsista PIBIC/CNPq, Graduanda em Licenciatura em Ciências Biológicas, Universidade Estadual de Feira de \\ Santana, e-mail: vaninemaia51@gmail.com \\ 2. Orientador, Departamento de Ciências Biológicas, Universidade Estadual de Feira de Santana, e-mail: \\ fbravo@uefs.br
}

PALAVRAS-CHAVE: Mantodea, Semiárido, Mata Atlântica

\section{INTRODUÇÃO}

Os integrantes da ordem Mantodea Burmeister, 1838 são conhecidos vulgarmente como louva-a-deus (louva-deus), põe-mesa (ou ponha mesa), pai-de-cobra, bendito e cavalinho-do-nosso-senhor (TERRA \& AGUDELO, 2012).

São conhecidas no mundo aproximadamente 2.400 espécies/subespécies de mantodeos (EHRMANN, 2002), e desse total apenas 20\% são encontrados na região Neotropical, o que corresponde aproximadamente a 498 espécies (RIVERA 2010). No Brasil existem registros de 244 espécies e 68 gêneros (AGUDELO, 2016). A riqueza estimada para o país em relação aos mantodeos é de 700 espécies (TERRA \& AGUDELO, 2012), ou seja, atualmente temos conhecimento de menos da metade de espécies dessa ordem no Brasil. No Semiárido brasileiro foram registradas todas as famílias brasileiras de Mantodea, 36 espécies e 26 gêneros (MENEZES \& BRAVO, 2013).

Este projeto visa desenvolver atividades com um grupo de insetos que possui grande representatividade no Museu de Zoologia da UEFS que precisam ser identificados (Figura 1). Além disso, existe a necessidade de discutir se uma delimitação de espécie mais ampla (lumper) ou mais estrita (splitter) pode ter sustento com análises de análises de séries grandes de uma espécie. Ainda tem como objetivo, ampliar o conhecimento sobre a fauna da Mata Atlântica e Semiárido.

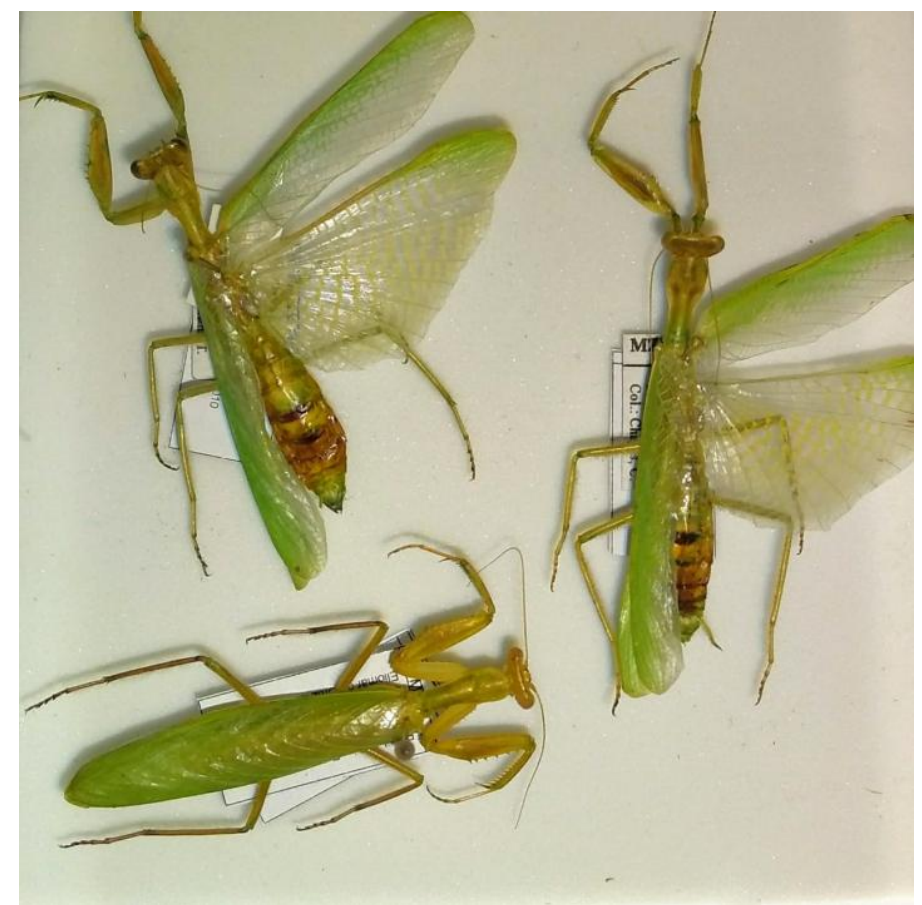

Figura 1 Margaromantis margaritaria, habitus dorsal, machos. 


\section{MATERIAL E MÉTODOS OU METODOLOGIA}

Foram estudados espécimes alfinetados a seco e armazenados em álcool 70\% procedentes de várias localidades do Nordeste brasileiro, depositados na Coleção Entomológica Professor Johann Becker do Museu de Zoologia da Universidade Estadual de Feira de Santana (MZFS). Os espécimes que foram coletados em localidades da Mata Atlântica foram posteriormente identificados e montados. Os exemplares em álcool 70\% foram preparados e montados em alfinete entomológico, seguindo a recomendação de AGUDELO \& CHICA (2002). Uma vez secos na estufa a 42 graus Celsius por ao menos sete dias, os espécimes foram etiquetados e tombados no Museu de Zoologia da UEFS (MZFS).

Para a identificação das espécies foram usadas as chaves de identificação para gênero de TERRA (1995), AGUDELO \& CHICA (2002) e chave para as espécies de GIGLIOTOS (1927).

As coletas foram feitas com redes entomológicas e busca ativa, além de armadilhas luminosas. Os espécimes foram sacrificados em câmaras mortíferas com acetato de etila e durante a coleta ativa, alguns foram criados em aquários com terra, permitindo assim o estudo de comportamento dos mesmos.

\section{RESULTADOS E/OU DISCUSSÃO}

No total, foram estudados e revisados aproximadamente 973 exemplares depositados na Coleção Entomológica Prof. Johann Becker do Museu de Zoologia da Universidade Estadual de Feira de Santana (MZFS). Grande parte desse material foi coletado em várias localidades do Semiárido brasileiro pelo projeto PPBio (Programa de Pesquisa em Biodiversidade).

No decorrer do trabalho foram identificados 870 espécies de seis famílias: Chaeteessidae, Mantoididae, Acanthopidae, Thespidae, Liturgusidae e Mantidae, sendo que a maior representação no museu são de espécies da família Mantidae (Figura 2), seguido por Thespidae. Dos 870 espécimes, 103 foram descritas até gênero, por conta de chaves confusas ou mesmo pela falta delas, visto que ainda é um grupo pouco estudado.

Após montagem e identificação do material, foi feita uma lista com o levantamento da quantidade e variedades de espécies presentes no museu, juntamente com suas respectivas localidades, apontando que a maioria do material é proveniente do Nordeste brasileiro, o que mostra a riqueza da região.

No ano de 2013 foi publicado o livro "Artrópodes do Semiárido. Biodiversidade e conservação" dos autores Freddy Bravo e Adolfo Calor, onde no capítulo 9 é apresentada uma lista com o número de espécies conhecidas e coletadas que estavam depositadas no MZFS. O presente trabalho também é um acréscimo para a coleção, visto que traz a atualização do material que se encontra no museu. 


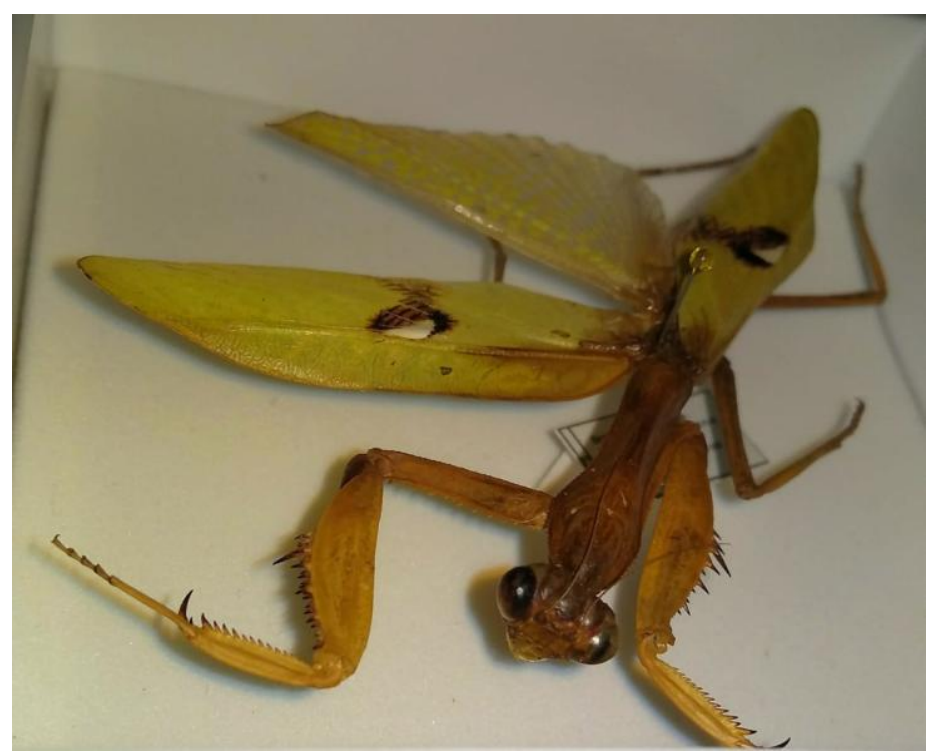

Figura 2 Stagmatoptera sp., habitus dorsal, macho

\section{CONSIDERAÇÕES FINAIS}

Dos 973 exemplares estudados e revisados, todos os espécimes de Mantodea foram identificados, sendo que cerca de 103 foram identificados até gênero pela ausência de chaves de identificação, e estão montados e depositados na coleção do MZFS.

Durante o levantamento e revisão feita do material, percebeu-se que a região Nordeste, com maior enfoque para o Semiárido, é muito rica em diversidade, visto que foram identificados espécies das cinco famílias existentes na região Neotropical.

Por ser um grupo restrito a poucos especialistas e por apresentarem acentuado dimorfismo, alguns espécimes ficaram identificados até gênero, porém mais da metade do material foi identificado até espécie.

\section{REFERÊNCIAS}

AGUDElO A.A 2018. Mantodea in Catálogo Taxonômico da Fauna do Brasil.

PNUD. Disponível em: 〈http://fauna.jbrj.gov.br/fauna/faunadobrasil/348 $>$.

Acesso em: 08 Ago. 2018.

AGUDELO, A. \& CHICA, L. 2002. Mantidos: Introducción al Conocimento del

Orden Mantodea. Universidad Distrital Francisco José de Caldas, Centro de Investigación y Desarrollo Científico, Bogotá, 90 p.

EHRMANN, R. 2002 Gottesanbeterinnen der Welt. Munster: Natur und Tier Verlag, $519 \mathrm{pp}$.

GIGLIO-TOS, E. 1927. Orthoptera-Mantidae. Das tierreich. Walter de Gruyter \& Co., Berlin, 50, 707p.

GRIMALDI, D.A. \& ENGEL, M.S. 2005. Evloction of the Insects. Cambridge University Press, New York, 784 pp. 
MENEZES, E. C. \& BRAVO, F, 2014. Capítulo 9. Mantodea (Insecta) do Semiárido. In Bravo. F \& Calor (orgs). Artrópodes do Semiárido: Biodiversidade e Conservação, Print Mídia. Feira de Santana. pp. 11-116.

MENEZES, E.C. \& F. BRAVO. 2013. Uma nova espécie de Orthoderella Giglio-Tos (Mantodea, Mantidae, Phontinainae) do Brasil. Revista Brasileira de Entomologia 57: 12-18.

RIVERA, J. 2010. A historical review of praying mantid taxonomy and systematics in the Neotropical Region: State of Knowledge and recente advances (Insecta: Mantodea). Zootaxa, 2638:44-64.

RODRIGUES, Henrique Miranda. Revisão taxonômica de Stagmatoptera Burmeister, 1838 (Mantodea, Mantidae, Stagmatopterinae). 2013. Dissertação (Mestrado em Sistemática, Taxonomia Animal e Biodiversidade) - Museu de Zoologia, Universidade de São Paulo, São Paulo, 2013. doi: 10.11606/D.38.2013.tde-11112013-181254. Acesso em 2018-07-20.

TERRA, P. S. \& AGUDELO, A. A. 2012. Mantodea, p.323-331. In: J.A. Rafael, G.A. R. Melo, C.J. B. De Carvalho, S.A. Casari \& R. Constantino (eds.). Insetos do Brasil. Diversidade e Taxonomia. Manaus, Holos Editora. 810p.

TERRA, P.S. 1995. Revisão Sistemática dos Gêneros de Louva-a-Deus da região Neotropical. (Mantodea). Revista Brasileira de Entomologia, 39(1), 13-94. 